\title{
Als Arzt in einer schwimmenden Praxis
}

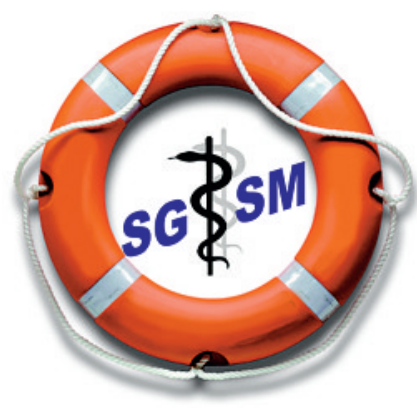

Kennen Sie die kleinste Arztpraxis in der Schweiz? Sie umfasst genau 10,5 Quadratmeter und bietet Platz für eine Liege, ein EKG-Gerät, einen Stuhl und einen Schreibtisch. An der linken Wand hängen zwei Glasschränke mit Notfallmedikamenten, medizinischen Instrumenten und Verbandsmaterial. Die Wand gegenüber schmückt ein gerahmtes Diplom, das den Praxisinhaber als Facharzt für Seemedizin auszeichnet. Geradeaus schaut man durch ein kleines, rundes Fenster aus Panzerglas, direkt aufs Wasser.

Denn diese Praxis befindet sich auf dem Bielersee, genauer gesagt auf der MS Berna, und ist der Arbeitsplatz des Kollegen Urs Schifferli. Sein Name könnte als Omen für die Erfüllung eines Lebenstraums gelten, nämlich den Arztberuf mit Reiselust zu verbinden. Seit Ostern 2008 übt Urs Schifferli seine Tätigkeit als einer von inzwischen drei Schiffsärzten auf dem Bielersee aus. Mehr noch: Er wurde der erste Titelträger des vor kurzem ins Leben gerufenen Facharztes für Seemedizin.

Insgesamt gibt es heute bereits 13 Fachärztinnen und -ärzte, die ihren Dienst bei der weissen Flotte auf den schweizerischen Binnenseen verrichten. Eine nahezu unglaubliche Entwicklung! Innerhalb kürzester Zeit wurde die neue Fachrichtung von der FMH akkreditiert. Allerdings waren auch erhebliche Hürden innerhalb der Ärzteschaft zu überwinden - die Schweizerische Ärztezeitung berichtete vor zwei Jahren ausführlich darüber $[1,2]$.

Wir erinnern uns: In der Herbstsession 2006 verabschiedete das Parlament das Gesetz über die Krankenfürsorge auf See, kurz GKrS. Mit Aufnahme des Schifffahrtbetriebes im Frühjahr 2008 trat es in Kraft. Schiffe mit mehr als 100 Personen an Bord mussten von nun an mit einem Schiffsarzt besetzt sein; bei mehr als 500 Personen besteht sogar die Auflage, einen zweiten Schiffsarzt mitreisen zu lassen. Da der Schiffsarzt gemäss GKrS ausreichende Kenntnisse für die ärztliche Tätigkeit auf See nachzuweisen hat, war die Schweizer Ärzteschaft gefordert, mit Hochdruck ein entsprechendes Weiterbildungsprogramm auszuarbeiten.

Am 1. April 2007 konstituierte sich die Schweizerische Gesellschaft für Seemedizin (SGSM), um sich dieser Herausforderung zu stellen, und zu Beginn des vergangenen Jahres - kurz vor Inkrafttreten des neuen Gesetzes - stand den ersten Anwärtern der Erwerb des neugeschaffenen Facharzttitels für Seemedizin offen. Neben guten allgemeinmedizinischen Kenntnissen gehören Erfahrungen als Notfallmediziner, Retkeit? Schweiz Ärztezeitung. 2007; 88:813-5. tungsschwimmer- und Tauchbescheinigungen sowie ein Seediensttauglichkeitsnachweis dazu. ${ }^{*}$

Die SGSM bietet entsprechende Kurse in $\mathrm{Zu}$ sammenarbeit mit der deutschen Seenotrettungsgesellschaft an und steht für alle Rückfragen rund «um das nasse Element» gerne zur Verfügung. Ja, aktiv ist die kleine Fachgesellschaft. Ihr derzeit grösstes Problem besteht darin, nicht mit der Schweizerischen Gesellschaft für Sportmedizin verwechselt $\mathrm{zu}$ werden; diese bezeichnet sich ebenfalls SGSM und hat sich bisher hartnäckig geweigert, eine Umbenennung vorzunehmen.

Die meisten Seemediziner haben bereits eine abgeschlossene Weiterbildung in Anästhesiologie oder Allgemeinmedizin. Auch Urs Schifferli ist eigentlich Anästhesist. Von November bis März arbeitet er als Oberarzt im Spitalzentrum Biel. Doch in der Schiffssaison behandelt er auf seiner MS Berna - wie die ersten Erfahrungen der Saison 2008 zeigen - hauptsächlich Patienten mit Platz- und Schnittwunden, Seekrankheit, Sonnenbrand und Menstruationsbeschwerden. «Denn viele unserer Schiffsgäste sind schliesslich weiblich», so der Seemediziner. Auch das Bordpersonal nimmt hin und wieder ärztliche Hilfe in Anspruch, in erster Linie wegen Schiffsneurosen. Einen Nebenverdienst wirft zudem der von ihm betriebene Bordshop ab, in dem z.B. Sonnencreme und Antiemetika erhältlich sind.

Schiffsarzt - ein Traumjob? Der Mann in Weiss, an der Reling stehend, Arztbriefe diktierend und sich von einer frischen Brise die Haare zerzausen lassend ... Mit dieser Art von Abenteuerromantik hat die Wirklichkeit eines Schiffsarztes allerdings wenig gemein. «An heissen Tagen kann in der Praxis schon mal die Hölle los sein», bestätigt Urs Schifferli. «Traumschiffatmosphäre spürt man dann sicherlich nicht.» Und trotzdem liebt er, genauso wie die anderen 12 Seemediziner, seinen Beruf und die mit ihm verbundene Freiheit. Zudem offerieren fast alle schweizerischen Schiffahrtgesellschaften eine kostenlose Verpflegung an Bord.

«Uns Seemedizinern geht es gut», fasst Urs Schifferli zusammen. Überzeugen Sie sich selbst: Einen authentischen Eindruck vom Leben und Arbeiten auf See gewährt die Fotogalerie auf der Homepage der Fachgesellschaft (www.sgseemed.ch).

Neugierige Kolleginnen und Kollegen dürfen unsere Schiffsärzte auch gerne einmal begleiten, um weitere Einblicke in deren Tätigkeit zu gewinnen. Schreiben Sie an seemedizin.info@gmx.ch, um einen Termin zu vereinbaren oder um mehr Informationen zu erhalten. 